\title{
Tricuspid Regurgitation in Ostium Secundum Atrial Septal Defects: Repair or Not?
}

\author{
Chirag Sumithra Prasanna Kumar, MCh, ${ }^{1}$ Bineesh K. Radhakrishnan, MCh, ${ }^{1}$ Remya Sudevan, MPH, ${ }^{2}$ \\ Jayakumar Karunakaran, $\mathrm{MCh}^{1}$
}

${ }^{1}$ Department of Cardiovascular and Thoracic Surgery, Sree Chitra Tirunal Institute for Medical Sciences and Technology, Thiruvananthapuram, Kerala, India; ${ }^{2}$ Department of Health Sciences Research, Amrita Institute of Medical Sciences, Kochi. Kerala, India

\section{ABSTRACT}

Background: Longstanding ostium secundum atrial septal defects lead to functional tricuspid regurgitation. Significant functional tricuspid regurgitation associated with left heart valve disease is addressed at the time of primary left heart valve surgery. In contrast, there is no global recommendation for tricuspid regurgitation associated with atrial septal defects. This study assesses changes in tricuspid regurgitation after isolated atrial septal defect closure.

Methods: Retrospectively, records were examined of 100 patients who underwent isolated ostium secundum atrial septal defect closure without tricuspid valve repair. Echocardiograms were done preoperatively and 3 days, 3 months, and 1 year after surgery. Data on tricuspid regurgitation status, right ventricle dimensions, and pulmonary artery hypertension status were collected and analyzed.

Results: After surgical closure, echocardiography showed a regression of tricuspid regurgitation to mild or less in $76 \%$ of patients at 3 days, $89 \%$ at 3 months, and $93 \%$ at 1 year. Severe pulmonary artery hypertension (32\% patients preoperatively) showed statistically significant regression: $14 \%$ at 3 days, $10 \%$ at 3 months, and $2 \%$ at 1 year. Preoperatively, the mean right ventricular internal diameter was $37.9 \mathrm{~mm}$, which decreased to $34 \pm 5.5 \mathrm{~mm}$ (mean \pm standard deviation) at 3 days, $32.3 \pm 5.3 \mathrm{~mm}$ at 3 months, and $31.3 \pm 5.4 \mathrm{~mm}$ at 1 year. It was also noted that regression favored patients who were $<25$ years old.

Conclusion: Tricuspid valve repair may not be required in patients with ostium secundum atrial septal defect with functional tricuspid regurgitation.

\section{INTRODUCTION}

Although atrial septal defects (ASDs) represent approximately $10 \%$ of all congenital heart disease, the condition often remains asymptomatic and unrecognized until adulthood [Dehghani 2012]. Recent studies have reported the

Received December 29, 2019; received in revised form March 4, 2020; accepted March 4, 2020.

Correspondence: Dr. Bineesh K. Radhakrishnan, Associate Professor, Department of CVTS, Sree Chitra Tirunal Institute for Medical Sciences and Technology, Thiruvananthapuram, Kerala, India; 9497017919 (e-mail: bineeshkrishna@gmail.com). incidence of ASD as 3 per 10,000 live births, with a female preponderance of 2:1 [Gatzoulis 1996]. The spectrum of ASDs comprises ostium secundum ASD (OSASD), sinus venosus ASD, ostium primum ASD, and coronary sinus type ASD. OSASD is the most common, accounting for $70 \%$ of all ASDs [Rosas 2004].

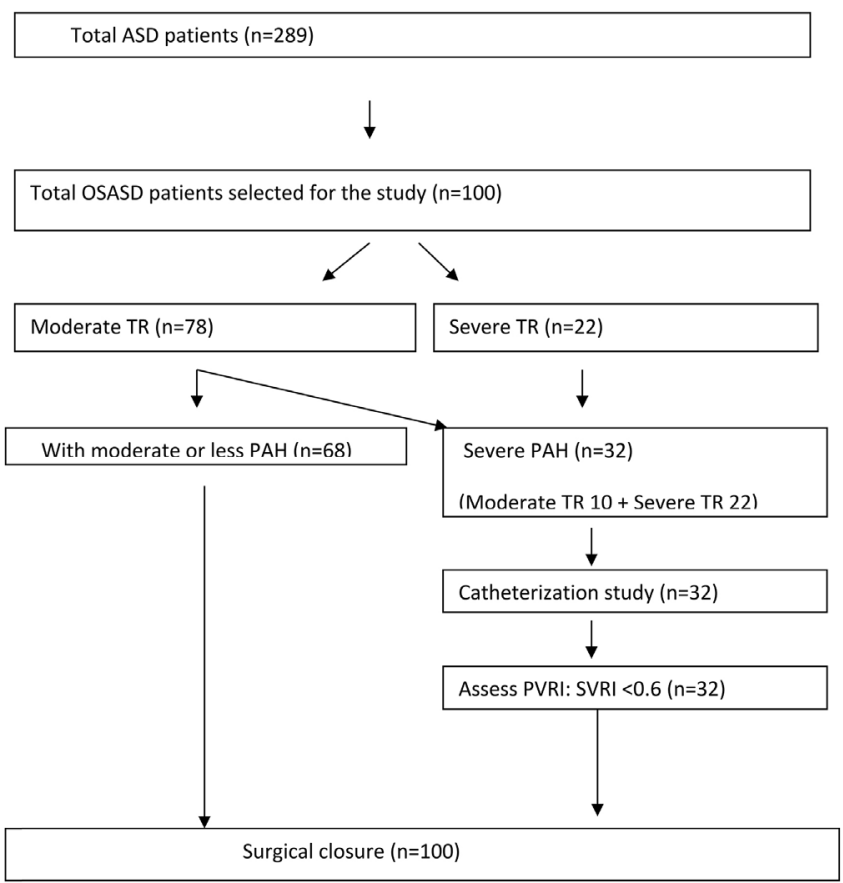

Figure 1. Patient selection flowchart.

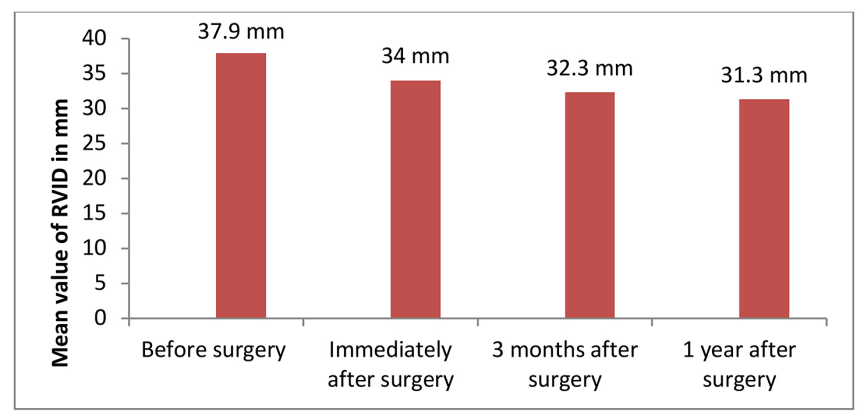

Figure 2. Comparison of RVID at different time points. 
Table 1. Comparison of Severity of Tricuspid Regurgitation at Different Time Intervals*

\begin{tabular}{|c|c|c|c|c|c|c|c|c|c|}
\hline TR & None & Trivial & Mild & Moderate & Severe & $Z$ versus Before Surgery & $P$ versus Before Surgery & $\chi^{2}$ & $P$ \\
\hline Before surgery & $0(0)$ & $0(0)$ & $0(0)$ & $78(78)$ & $22(22)$ & & & 215.45 & .000 \\
\hline 3 months after surgery & $25(25)$ & $28(28)$ & $36(36)$ & $7(7)$ & $4(4)$ & 8.45 & $<.001$ & & \\
\hline 1 year after surgery & $32(32)$ & $34(34)$ & $27(27)$ & $3(3)$ & $4(4)$ & 8.58 & $<.001$ & & \\
\hline
\end{tabular}

*Data are $\mathrm{n}(\%)$.

Table 2. Comparison of Pulmonary Artery Hypertension at Different Time Intervals*

\begin{tabular}{lccccc}
\hline PAH & None & Mild & Moderate & Severe & $\chi^{2}$ \\
\hline Before surgery & $2(2)$ & $39(39)$ & $27(27)$ & $32(32)$ & 157.3 \\
Immediately after surgery & $6(6)$ & $42(43)$ & $38(38)$ & $14(14)$ & .000 \\
3 months after surgery & $43(43)$ & $29(29)$ & $18(18)$ & $10(10)$ & \\
1 year after surgery & $52(52)$ & $26(26)$ & $20(20)$ & $2(2)$ & \\
\hline
\end{tabular}

*Data are n (\%).

Table 3. Comparison of Change in RVID from Baseline to Follow-Up, Stratified by Age Categories*

\begin{tabular}{|c|c|c|c|c|c|}
\hline RVID & Mean $(\mathrm{mm})$ & SD & Mean (mm) & SD & $\mathrm{P}$ \\
\hline Baseline & 35.13 & 3.338 & 39.80 & 5.330 & $<.001$ \\
\hline 3 days after surgery & 31.42 & 3.92 & 35.85 & 5.74 & $<.001$ \\
\hline 1 year after surgery & 28.26 & 3.78 & 33.39 & 5.34 & $<.001$ \\
\hline
\end{tabular}

Approximately $4 \%$ of ASDs tend to close spontaneously within 3 to 6 years, and thus early diagnosis gets neglected, with the expectation of spontaneous closure [Webb 2006]. Because of right ventricular (RV) compliance, the shunt is hemodynamically well tolerated, which keeps most patients asymptomatic until adulthood. The shunt volume across ASD depends on relative RV compliance, size of the defect, and atrial pressure difference. Long-term hemodynamic consequences are RV volume overload, pulmonary overcirculation, and pulmonary artery hypertension $(\mathrm{PAH})$, which end up in RV dilatation and hypertrophy. The process further stretches tricuspid annulus, leading to noncoaptation of leaflets and functional tricuspid regurgitation (TR). The majority of adult patients develop symptoms of reduced functional capacity and shortness of breath after the fourth decade of life in keeping with these hemodynamic changes [Krovetz 1972; Kotowycz 2013].
Echocardiography is the diagnostic modality of choice for quantification of ASD size and its hemodynamic consequences [Levin 1968]. ASD can be closed using devices by interventional cardiology techniques and surgical techniques [Moodie 2000; Rao 2017].

Both TR and PAH lead on to RV dysfunction in later stages [Oakley 1996]. The current guidelines for left heart valve lesions recommend concomitant tricuspid valve repair for patients with more than mild functional TR while operating for primary valve pathology [Nishimura 2014; Falk 2017]. The available guidelines are deficient in information regarding tricuspid valve repair for functional TR in ASD.

The aim of our study is to delineate the progress of TR in OSASD patients after surgical closure alone and without TR repair. The study also assesses the change in RV internal diameter (RVID) and PAH after surgical closure for a follow-up period of 1 year. 


\section{METHODS}

This is a hospital-based retrospective cohort study. Participants were OSASD patients with surgical closure alone (without tricuspid valve repair) from a tertiary care center. The study was approved by the institutional ethics committee. The inclusion criteria were patients aged $>12$ years having confirmed diagnosis of isolated OSASD with more than a mild degree of TR who had elective surgical closure alone; or OSASD patients with severe PAH who underwent cardiac catheterization and had a pulmonary vascular resistance:systemic vascular resistance (PVRI: SVRI) index $<0.6$ with elective surgical closure alone from January 2010 to January 2015. OSASD cases associated with complex congenital or other heart diseases (primary valvular pathology or coronary artery disease) requiring surgical corrections were excluded from the study. ASD closure with concomitant tricuspid valve procedures were also excluded. Patients who satisfied the selection criteria were enrolled for the study from the hospital information system (HIS). The duration of the study was 2 years. There were no dropouts in the follow-up period of 1 year.

Transthoracic echocardiogram readings, RVID, grade of $\mathrm{PAH}$, and severity of TR at 4 time points (preoperatively and 3 days, 3 months, and 1 year after surgery) were collected from the HIS. The reference limits and partition values of RVID, PAH clinical grading, and TR severity grading were determined according to previously established criteria (Appendix Tables A1, A2, and A3, respectively) [Lang 2005; Mohanan Nair 2012; Zoghbi 2003].

Privacy and confidentiality of patient details were maintained. Data were compiled and entered in Microsoft Excel, and statistical analysis was carried out using IBM Statistical Package for Social Science (SPSS version 17).

For statistical analysis, categorical and continuous variables were expressed as frequency (percentage) and mean \pm standard deviation, respectively. Comparison of TR and PAH at different time intervals was carried out using Friedman's test, and subgroup comparisons using Wilcoxon's signed rank test with Bonferroni correction. Comparison of RVID at different time intervals was carried out using repeated-measures analysis of variance and post hoc analysis using Bonferroni correction. Association of RVID with age was carried out using Mann-Whitney $\mathrm{U}$ test. $P<.05$ was considered the threshold for statistical significance.

\section{RESULTS}

A total of 100 patients who satisfied the selection criteria were enrolled for the study from the HIS. The patient selection flowchart is shown in Figure 1.

The mean age of the study population was $31.38 \pm 13.21$ years (range 13 to 65 ). There were 46 males $(46 \%)$ and 54 females (54\%), a male-to-female ratio of 0.85 . To evaluate the effect of surgery on age of presentation of OSASD with surgical correction, the patients were broadly grouped as $\leq 25$ years $(\mathrm{n}=38)$ and $>25$ years $(\mathrm{n}=62)$. Echocardiographic evaluations conducted on day 3 after surgery, 3 months, and 1 year were compared with preoperative echocardiography findings in terms of TR, PAH, and RV dimension.

\section{$T R$}

At the time of surgery, 78 patients $(78 \%)$ had moderate TR (3+), and $22(22 \%)$ had severe TR $(>3+)$ per echocardiographic preoperative evaluation. Immediate postoperative echocardiography showed regression of TR in $15 \%$ of patients to $0,31 \%$ to trivial, and $30 \%$ to mild, whereas $15 \%$ remained moderate $(3+)$ and $9 \%$ severe $(>3+)$. After 3 months of follow up, further regression in TR was noted, which showed $25 \%$ with TR $0,28 \%$ with TR $1+, 36 \%$ with TR $2+$, $7 \%$ with TR $3+$, and $4 \%$ with TR $>3+$. At the end of 1 year, $32 \%$ had regressed to TR $0,34 \%$ were TR $1+, 27 \%$ were TR $2+, 3 \%$ were $\mathrm{TR} 3+$, and $4 \%$ remained with $\mathrm{TR}>3+$. The subgroup comparison revealed significant regression of TR severity from baseline to all follow-up points (Table 1). Thus the rate of regression was high in the immediate postoperative period and continued to regress further at 3 months and 1 year.

\section{$P A H$}

Echocardiographic estimation of PAH before surgery was compared 3 days after surgery, 3 months, and 1 year. At the time of surgery, $32 \%$ of patients had severe PAH, $27 \%$ moderate, $39 \%$ mild, and $2 \%$ none. Immediately after surgery, only $14 \%$ still had severe $\mathrm{PAH}$; after 3 months just $10 \%$; and after 1 year, only $2 \%$ of patients had severe PAH $(P<.001$, Friedman's test and Wilcoxon's signed rank test). Even the comparison between 3 months and 1 year showed significant regression of PAH (Table 2).

\section{RVID}

RVID (millimeters) at the time of surgery using echocardiography as compared with measurements at 3 days, 3 months, and 1 year after surgery. Before surgery, RVID was $37.9 \mathrm{~mm}$; after 3 days, it was $34 \pm 5.5 \mathrm{~mm}$; at 3 months, $32.3 \pm$ $5.3 \mathrm{~mm}$; and at 1 year, $31.3 \pm 5.4 \mathrm{~mm}$ (Figure 2). A comparison of change in RVID with age is given in Table 3 .

Only 2 patients in our study series were in atrial fibrillation that was present preoperatively, and they continued to be on anticoagulation medication at last follow-up. All patients were asymptomatic except 4 had symptoms of New York Heart Association functional class II. None of the patients required surgical attention for tricuspid valve in the follow-up period.

\section{DISCUSSION}

Among congenital heart diseases, ASDs are common defects accounting of $10 \%$ of total defects. If not intervened at proper time, premature death can occur due to hemodynamic and electrophysiological complications. The optimal recommended treatment for ASDs is surgical closure, especially in the younger age group. A significant survival benefit is observed in surgically corrected patients compared with medically managed ones. 
Table A1. Reference Limits and Partition Values of Right Ventricular Size and Function as Measured in the Apical 4-Chamber View*

\begin{tabular}{lcccc}
\hline Parameter & Reference range & Mildly abnormal & Moderately abnormal & Severely abnormal \\
\hline RV diastolic area $\left(\mathrm{cm}^{2}\right)$ & 11 to 28 & 29 to 32 & 33 to 37 & $\geq 38$ \\
RV systolic area $\left(\mathrm{cm}^{2}\right)$ & 7.5 to 16 & 17 to 19 & 20 to 22 & $\geq 23$ \\
RV fractional area change $(\%)$ & 32 to 60 & 25 to 31 & $18-24$ & 1.0 to $1.2 \ddagger$ \\
TAPSE $(\mathrm{cm})$ & 1.5 to $2.0 \dagger$ & 1.3 to $1.5 \ddagger$ & $<17$ \\
\hline
\end{tabular}

*Data from Weyman 1994. TAPSE indicates tricuspid annular plane systolic excursion.

†Lang 2005.

†Hamer 1990.

Table A2. Clinical Grading of PAH by Echocardiography*

\begin{tabular}{lc}
\hline Grade of PAH & Right Ventricular Systolic Pressure (mmHg) \\
\hline Mild & 30.1 to 50 \\
Moderate & 50.1 to 70 \\
Severe & $>70$ \\
\hline
\end{tabular}

*Mohanan Nair 2012.

Table A3. Echocardiographic and Doppler Parameters Used in Grading Tricuspid Regurgitation Severity*

\begin{tabular}{|c|c|c|c|}
\hline Tricuspid valve & Usually normal & Normal or abnormal & Abnormal/flail leaflet/poor coaptation \\
\hline Jet area-central jets $\left(\mathrm{cm}^{2}\right)$ & $<5$ & 5 to 10 & $>10$ \\
\hline VC width $(\mathrm{cm})$ & Not defined & Not defined, but $<0.7$ & $>0.7$ \\
\hline Jet density and contour-CW & Soft and parabolic & Dense, variable contour & Dense, triangular with early peaking \\
\hline Hepatic vein flow & Systolic dominance & Systolic blunting & Systolic reversal \\
\hline
\end{tabular}

*Zoghbi 2003. CW indicates continuous wave Doppler; IVC, inferior vena cava; RA, right atrium; VC, vena cava.

The present study compared TR, RVID, and PAH before and after surgery in 100 OSASD patients of age $>12$ years. Our results suggest that there is significant regression in TR, RVID, and PAH at the end of 1 year after surgery without tricuspid valve repair.

RVID in the study group at the time of surgery and 1 year after surgery were $37.9 \pm 5.1$ and $31.3 \pm 5.4 \mathrm{~mm}$, respectively. The rate of regression in RV diameter was higher in patients who were younger ( $\leq 25$ years) versus older ( $>25$ years) in the follow-up from immediately after surgery to the end of 1 year. In a study comparing RVID regression in ASD patients before and after surgery, favorable regression in RVID was observed in patients $<25$ years old versus patients $>25$ [Ghosh 2007].
A comparative study on surgical closure and device closure of ASD for a 3-month follow-up in patients $>19$ years old found favorable regression in terms of $\mathrm{RV}$ end diastolic volume in their study group, using multi slice computed tomography volumetric analysis, which substantiates our study results of regression in $\mathrm{RV}$ dimension even with another imaging modality [Berbarie 2007]. We have not studied interventricular septal motion or cardiac catheterization evaluation, which might have enabled further elaborative comparison.

In our study group, $59 \%$ of patients had significant $\mathrm{PAH}$ at the time of surgery, and among them, $54.5 \%$ were $>30$ years of age. At the end of 1 year after surgery, only $22 \%$ of the patients still had significant PAH. In patients $>30$ years of age, only $4.5 \%$ still had severe PAH. Maatouk et al [2001], in their 
study on RV dilation after ASD closure, showed regression in $\mathrm{RV}$ dimension in the younger age group. Further imaging analysis and catheter-based study may be necessary to further evaluate $\mathrm{PAH}$ and $\mathrm{RV}$ dimension correlation, since the race of the selected study population and their genetic workup may represent significant variation in long-term postoperative outcomes [Maatouk 2001]. Liberthson et al [1981] showed regression in RV dimensions and improvement in functional class after ASD closure in patients with moderate to severe PAH, which parallels our study results, but they also stressed persistence or development of some degree of RV dysfunction in such patients. We were unable to analyze the progression of RV function because of limited follow-up time. Although $20 \%$ of patients had moderate PAH at the end of 1 year, all of them had functional class improvement. These patients need further follow-up to assess the clinical and echocardiographic features associated with $\mathrm{PAH}$, which is outside the scope of the present study.

In the current study, $78 \%$ of patients had moderate TR $(3+)$ and $22 \%$ severe TR $(>3+)$ at the time of presentation. At the end of 1-year follow-up, echocardiogram showed regression in TR among $93 \%$ of patients. The maximum regression was found in immediate postoperative period and in patients $<25$ years old. Gradual regression of TR was noted in patients $>25$ years old. At the end of 1 year, only $7 \%$ still had moderate or worse TR, with better functional status. Humenberger et al [2011] showed similar results, noting significant regression in $\mathrm{RV}$ dimension and reduction in $\mathrm{PAH}$, although their study group had only $12 \%$ of patients with significant TR before surgery. They also showed a decrease in degree of TR resulting from reverse remodeling of RV, along with improvement in functional status in all age groups undergoing ASD closure [Humenberger 2011]. Previous studies on quantitative assessment of pulmonary hypertension with TR by echocardiogram emphasized the functional nature of TR cases resulting from dilation of tricuspid annulus rather than intrinsic involvement of leaflets and pulmonary hypertension. Hence, the remodeling of RV due to PAH can culminate in significant TR [Humenberger 2011; Hoashi 2015].

Jemielity et al [2001] had better outcomes, such as a decrease in RV dimension and reduction in PAH and TR grade spontaneously after adult ( $>40$ years) ASD surgical closure. In spite of the better outcomes, tricuspid annuloplasty was needed in $27 \%$ of patients with TR, signifying the relevance of age [Jemielity 2001]. In the present study, all patients irrespective of age had decline in PAH, RV diameter, and TR grade after surgical correction without annuloplasty.

\section{Conclusion}

The results of the current study suggest that the progression of TR may be prevented by surgical closure alone. The other outcomes, such as RVID and PAH, were also better in all age groups at a follow-up of 1 year after surgical closure alone. The best outcomes were achieved in patients with less functional impairment and age $<25$ years. Considering the significant resolution of TR, RV remodeling, and PAH with surgical closure without a tricuspid valve repair, it may be recommended in all hemodynamically significant OSASDs irrespective of age and symptoms, immediately after diagnosis. This will help avoid an extra procedure and reduce hospital stay and schooldays or workdays lost, as well as lessen the financial burden. However, this study shows that PAH may not be resolved even if TR is reduced. We conclude that the current study should be considered a pilot phase for asserting the need of isolated ASD closure alone in OSASD patients having moderate or worse TR with or without PAH.

\section{Study Limitations}

This was a retrospective study with a limited number of study participants and short duration. There was no longterm follow up ( $>1$ year). Detailed preoperative and postoperative echocardiographic evaluation for annular diameter and leaflet morphology were not carried out.

\section{Recommendations}

A prospective comparative study should be conducted between isolated ASD closure and ASD closure with concomitant tricuspid repair, in a larger group of patients and for a longer duration. This will help in understanding more about the natural history of tricuspid regurgitation in ASD.

\section{REFERENCES}

Baumgartner H, Falk V, Bax JJ, et al. 2017.2017 ESC/EACTS Guidelines for the management of valvular heart disease. Eur Heart J 38:2739-2791.

Berbarie RF, Anwar A, Dockery WD, et al. 2007. Measurement of right ventricular volumes before and after atrial septal defect closure using multi slice CT. Am J Cardiol 99:1458-1461.

Dehghani H, Boyle AJ. 2012. Percutaneous device closure of secundum atrial septal defect in older adults. Am J Cardiovasc Dis 2:133-142.

Gatzoulis MA, Rodington AN, Somerville J et al. 1996. Should atrial septal defects in adults be closed? Ann Thorac Surg 61:657-659.

Ghosh S, Chatterjee S, Black E, Firmin RK. 2002. Surgical closure of atrial septal defects in adults: Effect of age at operation on outcome. Heart 88:485-487.

Hamer JPM. 1990. Practical Echocardiography in the Adult. Springer Netherlands, Dordrecht. 238 pp.

Hoashi T, Yazaki S, Kagisaki K, et al. 2015. Management of ostium secundum atrial septal defect in the era of percutaneous trans-catheter device closure: 7-Year experience at a single institution. J Cardiol 65:418-422.

Humenberger M Rosenhek R, Gabriel H, et al. 2011. Benefits of atrial septal defect closure in adults: Impact of age. Eur Heart J 32:553-560.

Jemielity M, Dyszkiewicz W, Paluszkiewicz L, et al. 2001. Do patients over 40 years of age benefit from surgical closure of atrial septal defects? Heart 85:300-303.

Kotowycz MA, Therrien J, Ionescu-Ittu R, et al. 2013. Long-term outcomes after surgical versus transcatheter closure of atrial septal defects in adults. JACC Cardiovasc Interv 6:497-503.

Krovetz LJ. Normal standards for cardiovascular data. II. Pressure and vascular resistances. 1972. John Hopkins Med J 130:187-195.

Lang RM, Bierig M, Devereux RB, et al. 2005. Recommendations for chamber quantification: A report from the American Society of Echocardiography's Guidelines and Standards Committee and the Chamber 
Quantification Writing Group, developed in conjunction with the European Association of Echocardiography, a branch of the European Society of Cardiology. Eur J Echocardiogr 7:79-108.

Levin AR, Levin AR, Spach MS, et al. 1968. Atrial pressure-flow dynamics in atrial septal defects (secundum type). Circulation 37:476-488.

Liberthson RR, Boucher CA, Strauss HW, Dinsmore RE, McKusick KA, Pohost GM. 1981. Right ventricular function in adult atrial septal defect: Preoperative and postoperative assessment and clinical implications. Am J Cardiol 47:56-60.

Maatouk F, Ben Farhat M, Betbout F, et al. 2001. Right ventricular dilatation and intraventricular septal motion after surgical closure of atrial septal defect. Arch Mal Coeur Vaiss 94:204-210.

Mohanan Nair KK, Pillai HS, Thajudeen A, et al. 2012. Immediate and long-term results following balloon mitral valvotomy in patients with atrial fibrillation. Clin Cardiol 35:35-39.

Moodie D, Sterba R. 2000. Long term outcomes excellent for atrial septal defect repair in adults. Cleve Clin J Med 67:657.

Nishimura RA, Otto CM, Bonow RO, et al. 2014. 2014 AHA/ACC
Guideline for the Management of Patients With Valvular Heart Disease: Executive summary: A report of the American College of Cardiology/ American Heart Association Task Force on Practice Guidelines. Circulation 129:2440-2492.

Oakley C. 1996. Closure of atrial septal defects in adult life. Cardiologia 41:31.

Rao PS, Harris AD. 2017. Recent advances in managing septal defects: Atrial septal defects. F1000Res 6:2042.

Rosas M, Attie F, Sandoval J, et al. 2004. Atrial septal defects in adults $\geq 40$ years old: Negative impact of low arterial saturation. Int J Cardiol $93: 145-155$

Webb G, Gatzolius MA. 2006. Atrial septal defects in the adult: Recent progress and overview. Circulation 114:1645-1653.

Weyman AE. 1994. Principles and Practice of Echocardiography, Second Edition. Lippincott Williams \& Wilkins; Philadelphia. 1335 pp.

Zoghbi WA, Enriquez-Sarano M, Foster E, et al. 2003. Recommendations for evaluation of the severity of native valvular regurgitation with two-dimensional and Doppler echocardiography. J Am Soc Echocardiogr 16:777-802. 